\title{
MINI-REVIEW
}

\section{Cannabinoids, Pain, and Opioid Use Reduction: The Importance of Distilling and Disseminating Existing Data}

\author{
Kent E. Hutchison, ${ }^{1, *}$ Sarah L. Hagerty, ${ }^{1}$ Jeffrey Galinkin, ${ }^{2}$ Angela D. Bryan, ${ }^{1}$ and L. Cinnamon Bidwell ${ }^{3}$
}

\begin{abstract}
The high prevalence of chronic pain conditions combined with an over-reliance on opioid prescriptions has resulted in an opioid epidemic and a desperate need for solutions. There is some debate about whether cannabis might play a role in addressing chronic pain conditions as well as the opioid epidemic. Recent surveys suggest that a large number of people are using cannabis as a treatment for pain and to reduce use of opioids, and cannabis-derived products demonstrate at least modest efficacy in the treatment of pain in randomized controlled trials. In addition, surveillance studies from countries that have approved the use of Sativex, which is a cannabis-based product, have demonstrated that a combination of $\Delta$-tetrahydrocannabinol and cannabidiol has low potential for harm, is well tolerated, and is helpful to patients. Given the number of people in the United States who are already using cannabis to manage pain and opioid use in state-regulated markets, it is imperative to conduct additional research in these areas, and to disseminate information on how to minimize harm and maximize any benefits of using cannabinoids to mitigate pain and reduce opioid use. The purpose of this article is to call attention to the fact that cannabis is being used in the management of chronic pain. Thus, this article also provides a set of guidelines on how to approach using cannabis to treat pain.
\end{abstract}

Keywords: cannabis; pain; opioids

As the nation continues to grapple with the interconnected problems of chronic pain and the opioid epidemic, practical, and effective solutions that can be deployed quickly remain elusive. Some reviews have suggested that cannabinoids may play an important role in both pain control ${ }^{1}$ and the opioid problem, ${ }^{2}$ while others have dismissed this possibility. ${ }^{3}$ A flaw among some of the more negative reviews is that they often ignore evidence from other parts of the world, indicating that a cannabis-derived medication (e.g., Sativex) is both effective at managing chronic pain and has very low potential for harm. Despite this evidence, there are currently no published guidelines on how to minimize harms and maximize benefits when using cannabis products. The intention of the present communication is to (1) call attention to the fact that many people are currently using cannabis to treat chronic pain and reduce opioid use; (2) advocate for research at the intersection of cannabis, pain, and opioids; and (3) provide preliminary evidence-based guidelines for using cannabis in light of the fact that many people are already choosing to do in the absence of such direction. To that end, we provide a brief synopsis of the twin problems of chronic pain and opioid dependence, followed by reviews of studies that have examined the effect of cannabis products on pain, and of those that have examined the effect of cannabis on opioid use. In addition, we summarize existing evidence on the safety and tolerability of different oral formulations of cannabis, and suggest evidence-based guidelines regarding the use of cannabis products in the management of pain and reduction of opioid use.

${ }^{1}$ Department of Psychology and Neuroscience, University of Colorado Boulder, Boulder, Colorado.

${ }^{2}$ Pediatric Anesthesiology, University of Colorado School of Medicine, Aurora, Colorado.

${ }^{3}$ Institute of Cognitive Science, University of Colorado Boulder, Boulder, Colorado. 


\section{Chronic Pain and the Opioid Epidemic}

The opioid epidemic is linked to the use of prescription opioids to manage pain, and recent estimates suggest that 25.3 million U.S. adults suffer from daily chronic pain. ${ }^{4}$ While there are a number of nonopioid-approved treatments for both neuropathic and somatic pain (e.g., nonsteroidal anti-inflammatory drugs (NSAIDS), tricyclic antidepressants, gabapentin, pregabalin), many of these treatments have demonstrated low efficacy, undesirable side effects, and poor tolerability. ${ }^{5,6}$ Further, adjunct therapies such as physical therapy, cognitivebehavioral treatments, and acupuncture, which are accepted as effective for chronic pain, ${ }^{7,8}$ are not always reimbursed by insurance companies. ${ }^{9}$

Over the past two decades, opioid prescriptions have risen dramatically. Between 1999 and 2010, there was a fourfold increase in opioid prescriptions, ${ }^{10}$ while the rate of opioid drug overdose deaths increased 200\% since the year $2000 .{ }^{11}$ Opioid-related overdose deaths continue to increase as those who were initially prescribed opioid medications have turned to street forms of opioids, including heroin and dangerously strong synthetics (i.e., fentanyl and fentanyl derivatives ${ }^{12}$ ). Although there are likely multifactorial reasons why this transition occurs, results from qualitative studies suggest that people who previously used prescription opioids transitioned to illegal forms of opioids due to cost and ease of access after becoming physically and emotionally addicted to prescription opioid pills. ${ }^{13}$ Some estimates suggest that among heroin injectors, 39\% report being "hooked on" prescription type opioids before transitioning to heroin. ${ }^{14}$

In addition to the high risk of overdose, there are a number of other concerns associated with long-term opioid use. For example, there is a distinct lack of evidence for the usefulness and safety of using opioids in the treatment of chronic pain conditions. ${ }^{15}$ Of the estimated 91.8 million U.S. adults who use prescription opioids for pain, $12.5 \%$ report misuse. ${ }^{16}$ The high rate of misuse could be explained, at least in part, by tolerance that develops with continued opioid use. Continued opioid use is associated with two key processes: opioid tolerance and opioid-induced pain sensitivity (hyperalgesia). In concert, these phenomena can reduce the efficacy of opioids for treating chronic pain and contribute to opioid misuse. ${ }^{17}$ Other studies suggest that opioid-induced central immune signaling underlies opioid tolerance and hyperalgesia. ${ }^{18}$ Relatedly, prolonged opioid use has been associated with neuroinflammation and damage to the brain and immune system. ${ }^{19}$ Finally, recent evidence suggests that opioid treatment for chronic pain is no more effective than nonopioid medication at facilitating better pain-related function. ${ }^{20}$ Therefore, in many cases, the adverse consequences associated with opioid use outweigh any benefit to the patient.

Taken together, the use of opioids as a long-term treatment for chronic pain is associated with notable drawbacks and distinct danger from a public health perspective. As a result, in 2016 the CDC published new guidelines regarding the prescription of opioids, including a set of 12 recommendations. ${ }^{21}$ While changes prompted by these guidelines represent important controls, they could compound the problem, as individuals who are dependent and/or rely on opioids to control pain might turn in increasing numbers to opioids purchased illegally, leading to more overdose deaths in the short term. ${ }^{22,23}$ In conclusion, while opioids are effective short-term analgesics, they have a negative long-term impact on the individual and on society. The United States needs new, effective approaches that address both pain management and opioid dependence.

\section{Pain Management and Cannabinoids}

Patient preferences and behavior

In recent years, a number of epidemiological studies and randomized controlled clinical trials have suggested that people are trying cannabis to treat pain conditions. For example, studies indicate that relief from chronic pain is by far the most common motivation among individuals who use medical cannabis, with $87-94 \%$ of medical cannabis users reporting that they use cannabis for relief of a pain condition. ${ }^{24,25}$ Existing data further suggest that people find cannabis to be an effective strategy for pain management. For example, in a recent study of 2897 medical cannabis patients in the United States, $>80 \%$ reported that cannabis was more effective than opioid medications for pain management. ${ }^{26}$ In another study, the authors surveyed 501 perioperative patients at Mt. Sinai Hospital, and found that $>80 \%$ believed that cannabis would help with pain management. ${ }^{27}$ Other studies ranging from survey studies among large community samples to open-label studies of medicinal cannabis have also suggested that many chronic pain patients effectively use cannabis to control pain. ${ }^{28-31}$ Thus, even in the absence of a consensus in the medical community that cannabinoids are effective at controlling pain, patients believe that cannabis helps control pain and are using cannabis to control pain. 
The conclusions drawn from the above studies should be interpreted in the context of the relevant limitations. Specifically, it should be acknowledged that many of the studies supporting the idea that cannabis is associated with decreased opioid use include survey/self-report data and time-series data at the state level. Randomized trials that test the question of whether cannabis use decreases opioid use are lacking. Therefore, additional research that more rigorously extends upon the existing literature needs to be done before concluding that a causal relationship exists between cannabis use and opioid use.

\section{Randomized controlled trials of cannabinoids and pain}

Systematic reviews of the available randomized controlled trials by the National Academy of Sciences and others have concluded that cannabinoids and cannabis products can be an effective tool with respect to the management of chronic pain. ${ }^{1,32-34}$ For example, 22 of 29 primary studies included in a systematic review by Lynch and Ware indicated that cannabinoids demonstrated at least modest efficacy and were a safe alternative for pain management. ${ }^{32}$ Importantly, all of these studies included either an active comparator or a placebo control. While there is a high degree of consistency in the direction of the effect, the estimate of the size of the effect for the effectiveness of cannabinoids varies from randomized controlled trial (RCT) to RCT. Variability in the estimates of effect size is not surprising given differences across studies, such as the clinical characteristics of the patients who were included, the types of cannabinoids that were included (e.g., plantderived, synthetic, THC, THC $+\mathrm{CBD}$ ), and the routes of administration (e.g., oral, oral mucosal, inhaled). Despite the differences in estimates of effect sizes across studies, the critical point is that the direction of the effect (i.e., that cannabinoids are at least modestly effective) is consistent across the majority of studies. $^{32}$ Of note is the fact that there are no published randomized clinical trials in which cannabis is substituted for opioids among patients misusing opioids for treatment of chronic pain. ${ }^{35}$

\section{Cannabinoids and Opioids}

Given evidence that patients believe that cannabinoids are an effective tool for pain management and objective evidence from RCTs that cannabinoids are effective in terms of controlling pain, a logical next question is whether there is evidence that individuals are actively substituting cannabinoids for opioids in the management of pain. In a large survey of patients $(n=2897)$, $97 \%$ of respondents "strongly agreed/agreed" that they are able to decrease the amount of opioids they consume when they also use cannabis. ${ }^{26}$ Similarly, a retrospective study found that medical cannabis use was associated with a $64 \%$ decrease in opioid use and improved ratings of quality of life. ${ }^{36}$ The "substitution effect" whereby patients appear to be using cannabis in place of opioids is also consistent with an open-label, prospective trial, which found that cannabis was associated with less opioid use, as well as reduced pain and improved quality of life. ${ }^{30}$

It is likely that survey studies and open-label trials suggesting that cannabis use is associated with decreases in opioid use may be biased by patient expectations. However, supporting the findings above, there is also robust pre-clinical evidence demonstrating that $\Delta 9$ tetrahydrocannabinol (THC), a cannabinoid receptor type 1 (CB1) agonist, increases the analgesic potency of opioids by eliciting additive and synergistic effects across routes of administration and species. ${ }^{37,38}$ This opioid-sparing effect of THC means that lower opioid doses are needed for effective analgesia, and that both adverse effects and reinforcing effects from opioids are reduced. ${ }^{39}$ Cannabidiol (CBD), which has a low affinity for the $\mathrm{CB} 1$ receptor and does not produce intoxication, produces pain-blocking effects in animal models of neuropathic pain, ${ }^{40-42}$ and may act to increase the effect of opioids on reducing acute pain. ${ }^{43} \mathrm{CBD}$ may also diminish abuse-related effects of opioids and THC. ${ }^{44-47}$ These findings suggest that cannabinoids may enhance opioid analgesia while decreasing its abuse-related end-points.

There is also supporting evidence from studies that have examined opioid use through prescription data that are consistent with the findings described above. For example, one recent study examined opioid prescription records from a New Mexico state Prescription Monitoring Program to examine the effect of cannabis on opioid cessation (defined as the absence of opioid prescriptions) and opioid reduction (calculated in average daily morphine equivalents ${ }^{48}$ ). Using the prescription data, the results indicated that individuals who enrolled in the New Mexico Medical Cannabis Program were significantly more likely to quit or reduce using opioids than a control group of patients who were not enrolled in the cannabis program, and that those in the cannabis program reported greater increases in quality of life. Recent analyses of large pools of prescription data in states with access to 
medical cannabis also suggest a significant reduction in the filling of prescriptions for traditional pain medications. ${ }^{49}$ Most recently, a study used Multiple Cause of Death files available through the Centers for Disease Control and Prevention WONDER (Wide-Ranging Online Data for Epidemiologic Research) system to examine opioid-related deaths in Colorado before and after cannabis legalization. Findings suggest that legalization of cannabis was associated with a reduction of 0.7 opioid-related deaths per month. ${ }^{50}$ Thus, data from multiple sources suggest that cannabis may be associated with a reduction in opioid use and managing pain.

Although there are several studies supporting the promise of cannabis on reducing pain and opioid use, one recent observational study did not find any effect of cannabis on pain and opioid use. ${ }^{51}$ In addition, other prominent researchers have raised questions as to whether physicians should recommend replacing opioids with cannabis. ${ }^{35}$

\section{Safety and Tolerability of Cannabis in Various Formulations}

Health care providers are often reluctant to discuss the use of cannabis products with their patients and cite the lack of studies regarding (1) safety and tolerability, (2) efficacy in general and efficacy of specific formulations, and (3) dosing information and guidelines. In fact, a survey of 532 family health care providers in Colorado indicated that only $31 \%$ had ever recommended cannabis products to patients and $71 \%$ of those health care providers had recommended it to $<5$ patients. ${ }^{52}$ One reason for this may be that cannabis use has some documented adverse side effects and tolerability concerns that could complicate its utility in treating pain. One meta-analysis of the efficacy and tolerability of cannabis products for chronic pain suggests that cannabis is associated with a variety of adverse events and side effects, including mood disturbance, disassociation/acute psychosis, dizziness/drowsiness, and cognitive problems. ${ }^{53}$ In addition, some side effects might be pronounced among patient populations who are experiencing psychiatric disorders or symptoms. For example, cannabis use has been associated with increased levels of depression and anxiety among patients who were experiencing elevated symptoms of depression and being treated in a psychiatric outpatient setting. ${ }^{54}$ Importantly, these adverse events and side effects might differ depending on the type and chemovar of cannabis. For example, cannabis products with higher ratios of CBD:THC are associated with fewer cognitive and psychiatric negative consequences. ${ }^{55}$

While it is true that there have been very few studies on efficacy, safety, and tolerability in the United States, pharmaceutical companies have been actively developing and testing plant-derived cannabis compounds that contain both THC and CBD in Europe, Canada, and Israel for some time. Specifically, Sativex is a plant-derived cannabis medication that consists of approximately equal parts of THC and CBD and is administered orally through a spray. Sativex has recognized analgesic effects, ${ }^{56-58}$ and has been approved for use in the United Kingdom, Canada, and 28 countries in Europe, South America, and Asia.

One of the potential side effects of Sativex that was evaluated very carefully was the potential for abuse. Importantly, studies of adverse events related to the abuse potential of Sativex suggest that few $(\sim 2.2 \%)$ patients experience intoxication, euphoria, tolerance, or withdrawal. ${ }^{59-62}$ Consistent with these data, Sativex is currently a Schedule IV (Part 1) controlled drug in the United Kingdom, due to its low potential for abuse and diversion. Schedule IV in the United Kingdom is similar to Schedule IV in the United States. One putative mechanism that may explain an even lower potential for harm appears to be the combination of CBD with THC in Sativex. In fact, studies have suggested that CBD may attenuate the effects of THC on cognitive processing as well as on mood. ${ }^{63-66}$ Thus, a 1:1 formulation of THC plus $\mathrm{CBD}$ may mitigate some of the deleterious effects of THC.

Because Sativex has been approved for use in Canada since 2005 and the European Union (EU) since 2010, there are now postmarketing data that provide additional evidence regarding the safety and tolerability of a cannabis-derived medication with equal parts of THC to CBD. Global exposure to Sativex since it was marketed in the EU was estimated to be $>45,000$ patient years in 2016. ${ }^{67}$ After approval in 2010, a formal registry was implemented in the United Kingdom, Germany, and Switzerland to provide data on safety and tolerability, which includes 941 patients with 2213 patient years of exposure. The mean number of days of use was 954 days, and the mean daily dose across all patients was $14.6 \mathrm{mg}$ of THC and $13.5 \mathrm{mg}$ of CBD. In terms of perceived benefits, $83 \%$ reported that they benefited from the medication, and no long-term adverse cognitive effects were noted. A smaller registry in Spain reported results consistent with the larger study. ${ }^{68}$ Thus, both studies provide data consistent 
with past RCTs on safety and tolerability, ${ }^{60}$ suggesting that Sativex is safe and well tolerated with low abuse potential. $^{67,68}$

While data on plant-derived, oral formulations (i.e., edible or pill form) similar to Sativex with equal amount of THC and CBD that are now widely available in state-regulated markets in the United States are lacking, these formulations will likely have a profile highly similar to Sativex. While Sativex is an oral spray that is partially absorbed through mucosa, a direct comparison of Sativex with a formulation of oral THC without mucosal absorption indicated no significant differences in plasma pharmacokinetics. ${ }^{69}$ In addition, studies of serious adverse events related to formulations other than Sativex are broadly consistent with the studies on the safety profile of Sativex. ${ }^{70}$ Thus, it is likely that oral formulations combining $\mathrm{THC}$ and $\mathrm{CBD}$, which are commonly available in state-regulated markets, will perform similarly to Sativex.

\section{Conclusions and Guidelines}

In summary, retrospective studies suggest that many individuals have already turned to cannabis to alleviate pain and are already substituting cannabis for opioids. Systematic reviews of RCTs have indicated that cannabis products demonstrate efficacy in the management of pain. ${ }^{1,32-34}$ RCTs, patient registries, and the experience of 30 countries that have approved a cannabis plant-derived product that is equal parts of THC to CBD (i.e., Sativex) indicate that this product is well tolerated with low potential for harm. ${ }^{60,67,68}$ Subjective and objective data suggest that individuals who use cannabis for pain are able to decrease their use of opioids and potentially other medications. ${ }^{26,71}$ These findings provide an empirical basis for the role of cannabis in pain management and the opioid epidemic.

In the United States, half of the population and more than half of our aging population are actively looking for strategies to manage pain. Meanwhile, the opioid epidemic is a major public health threat. These realities combined with the shifting legal and political landscape in which $>20 \%$ of the nation now has access to stateregulated cannabis suggest that guidance is needed from scientists or policy makers for the millions of Americans who will turn to cannabis products over the next several years. In this context, it is a disservice to the public not to provide evidence-based guidelines that are intended to enhance public health and safety. Unbiased information, education, and communication about the potential benefits and potential risks of can- nabis products are sorely needed. While the evidence base is far from mature, there is sufficient evidence to develop and disseminate some basic information to the public and to the medical community. To that end, specific guidelines are provided below. These guidelines will change as the evidence base continues to develop.

\section{Clinical Guidelines Based on Existing Evidence}

Based on the evidence presented above, we propose several evidence-based guidelines for patients who have already decided to use cannabis products for pain and/or to reduce opioid use as well as for health care providers who have decided to discuss these options with their patients.

(1) Oral formulations (e.g., pill, edible, oromucosal) that have equal amounts of THC and CBD are likely to have some benefits and minimal risks, consistent with the data on Sativex in Canada, Europe, and elsewhere.

(2) Although doses vary widely, studies suggest that the average THC dose individuals use for medical purposes is $3 \mathrm{mg}$ daily. ${ }^{72}$ Further, based on the dosing regimen for Sativex and research indicating that other oral formulations have a highly similar pharmacokinetic profile, ${ }^{61}$ patients may achieve therapeutic benefit and minimal psychoactivity with oral doses as low as $2.5 \mathrm{mg}$ THC. $^{73}$

(3) If needed, patients can then work with their physician to slowly increase the THC dose by $2.5 \mathrm{mg}$ until the desired effect (i.e., reduction in pain, reduction in opioid use) is achieved. Careful monitoring is critical with oral THC formulations, as patients may be at risk of taking more than the intended dose. Note CBD dosing can start higher and progress faster as it is not psychoactive, with careful physician monitoring at high doses for potential pharmacokinetic interactions. It is also critical that all cannabis products be kept out of reach of children.

(4) Patients should avoid products that have not been manufactured in cGMP compliant facilities and have accurate dosing information on the label. To that end, state regulatory officials should provide information to the public regarding the accuracy of labeling using data from reputable laboratories to verify the dose of cannabinoids in products available in state-regulated markets. 
While this has increasingly become the case as states have adopted more stringent regulatory practices, studies suggest that many products are not labeled accurately. ${ }^{74}$ Labeling accuracy needs to be addressed at the state level. In addition, patients should be made aware of the variation in state medical marijuana programs regarding regulatory practices. $^{75}$

(5) In cases where patients experience unwanted cognitive side effects, patients should discontinue use or switch to products with greater levels of CBD and lower levels of THC, as this might reduce deleterious cognitive and moodrelated consequences. ${ }^{55}$

(6) The risk and benefits of cannabis use should be evaluated carefully in some individuals because the risks of using cannabis may outweigh the benefits for people who

a. are under the age of 25

b. have a personal history or strong family history of psychosis

c. are actively treated for or are symptomatic with mood/depressive and anxiety disorders

d. have a current or past cannabis use disorder or another active substance use disorder

e. have cardiovascular or respiratory disease

f. are pregnant, planning to become pregnant, or are breastfeeding.

\section{Author Disclosure Statement}

No competing financial interests exist.

\section{References}

1. National Academies of Sciences E. The Health Effects of Cannabis and Cannabinoids: the Current State of Evidence and Recommendations for Research [Internet]. 2017 [cited 2018 Mar 7]. Available at: https:// www.nap.edu/catalog/24625/the-health-effects-of-cannabis-andcannabinoids-the-current-state (last accessed on April 24, 2019).

2. Hurd YL. Cannabidiol: swinging the marijuana pendulum from 'weed' to medication to treat the opioid epidemic. Trends Neurosci. 2017; 40:124-127.

3. Christie GC, Baker CGC, Cooper GR, et al. The President's commission on combating drug addiction and the opioid crisis. WhiteHouse Gov, 2017.

4. Nahin RL. Estimates of pain prevalence and severity in adults: United States, 2012. J Pain. 2015;16:769-780.

5. Kroenke K, Krebs EE, Bair MJ. Pharmacotherapy of chronic pain: a synthesis of recommendations from systematic reviews. Gen Hosp Psychiatry. 2009;31:206-219.

6. Turk DC. Clinical effectiveness and cost-effectiveness of treatments for patients with chronic pain. Clin J Pain. 2002;18:355-365.

7. Thomas D-A, Maslin B, Legler A, et al. Role of alternative therapies for chronic pain syndromes. Curr Pain Headache Rep. 2016;20:29.

8. Cherkin DC, Sherman KJ, Balderson BH, et al. Effect of mindfulness-based stress reduction vs cognitive behavioral therapy or usual care on back pain and functional limitations in adults with chronic low back pain: a randomized clinical trial. JAMA. 201622;315:1240-1249.

9. Pelletier KR, Astin JA. Integration and reimbursement of complementary and alternative medicine by managed care and insurance providers:
2000 update and cohort analysis. Altern Ther Health Med. 2002;8:38-39, 42, 44 passim.

10. Centers for Disease Control and Prevention (CDC). Vital signs: overdoses of prescription opioid pain relievers-United States, 1999-2008. MMWR Morb Mortal Wkly Rep. 2011;60:1487-1492.

11. Rudd RA, Aleshire N, Zibbell JE, et al. Increases in drug and opioid overdose deaths-United States, 2000-2014. Am J Transplant. 2016;16: 1323-1327.

12. Schuchat A, Houry D, Guy GP. New data on opioid use and prescribing in the United States. JAMA. 2017;318:425-426.

13. Mars SG, Bourgois P, Karandinos G, et al. "Every 'Never' I Ever Said Came True": transitions from opioid pills to heroin injecting. Int J Drug Policy. 2014;25:257-266.

14. Peavy KM, Banta-Green CJ, Kingston S, et al. "Hooked on" prescriptiontype opiates prior to using heroin: results from a survey of syringe exchange clients. J Psychoactive Drugs. 2012;44:259-265.

15. Chou R, Deyo R, Devine $B$, et al. The effectiveness and risks of long-term opioid treatment of chronic pain. Agency for Healthcare Research and Quality; US, 2014.

16. Han B, Compton WM, Blanco C, et al. Prescription opioid use, misuse, and use disorders in U.S. adults: 2015 National Survey on Drug Use and Health. Ann Intern Med. 2017;167:293.

17. Chang G, Chen L, Mao J. Opioid tolerance and hyperalgesia. Med Clin. 2007;91:199-211.

18. Grace PM, Maier SF, Watkins LR. Opioid-induced central immune signaling: implications for opioid analgesia. Headache. 2015;55:475-489.

19. Watkins LR, Hutchinson MR, Rice KC, et al. The "toll" of opioid-induced glial activation: improving the clinical efficacy of opioids by targeting glia. Trends Pharmacol Sci. 2009;30:581-591.

20. Krebs EE, Gravely A, Nugent S, et al. Effect of opioid vs nonopioid medications on pain-related function in patients with chronic back pain or hip or knee osteoarthritis pain: the SPACE randomized clinical trial. JAMA. 2018;319:872-882.

21. Dowell D, Haegerich TM, Chou R. CDC guideline for prescribing opioids for chronic pain-United States, 2016. JAMA. 2016;315:1624-1645.

22. Weiss RD, Potter JS, Fiellin DA, et al. Adjunctive counseling during brief and extended buprenorphine-naloxone treatment for prescription opioid dependence: a 2-phase randomized controlled trial. Arch Gen Psychiatry. 2011;68:1238-1246.

23. Weiss RD, Potter JS, Griffin ML, et al. Long-term outcomes from the national drug abuse treatment clinical trials network prescription opioid addiction treatment study. Drug Alcohol Depend. 2015;150:112-119.

24. Ilgen MA, Bohnert K, Kleinberg F, et al. Characteristics of adults seeking medical marijuana certification. Drug Alcohol Depend. 2013;132: 654-659.

25. Light MK, Orens A, Lewandowski B, et al. Market size and demand for marijuana in Colorado. Colorado Department of Revenue. 2014. Available at: https://www.colorado.gov/pacific/sites/default/files/ Market\%20Size\%20and\%20Demand\%20Study,\%20July\%209,\%202014 (last accessed on April 24, 2019).

26. Reiman A, Welty M, Solomon P. Cannabis as a Substitute for OpioidBased Pain Medication: patient Self-Report. Cannabis Cannabinoid Res. 2017;2:160-166.

27. Khelemsky Y, Goldberg AT, Hurd YL, et al. Perioperative patient beliefs regarding potential effectiveness of marijuana (cannabinoids) for treatment of pain: a prospective population survey. Reg Anesth Pain Med. 2017;42:652-659.

28. Abrams DI, Couey $\mathrm{P}$, Shade SB, et al. Cannabinoid-opioid interaction in chronic pain. Clin Pharmacol Ther. 2011;90:844-851.

29. Degenhardt L, Lintzeris N, Campbell G, et al. Experience of adjunctive cannabis use for chronic non-cancer pain: findings from the Pain and Opioids IN Treatment (POINT) study. Drug Alcohol Depend. 2015; 147:144-150.

30. Haroutounian S, Ratz Y, Ginosar Y, et al. The effect of medicinal cannabis on pain and quality-of-life outcomes in chronic pain: a prospective open-label study. Clin J Pain. 2016;32:1036-1043.

31. Sznitman SR, Bretteville-Jensen AL. Public opinion and medical cannabis policies: examining the role of underlying beliefs and national medical cannabis policies. Harm Reduct J. 2015;12:46.

32. Lynch ME, Ware MA. Cannabinoids for the treatment of chronic noncancer pain: an updated systematic review of randomized controlled trials. J Neuroimmune Pharmacol. 2015;10:293-301. 
33. Lynch ME, Campbell F. Cannabinoids for treatment of chronic non-cancer pain; a systematic review of randomized trials. Br J Clin Pharmacol. 2011;72:735-744.

34. Whiting PF, Wolff RF, Deshpande S, et al. Cannabinoids for medical use: a systematic review and meta-analysis. JAMA. 2015;313:2456-2473.

35. Humphreys K, Saitz R. Should physicians recommend replacing opioids with cannabis? JAMA. 2019;321:639-640.

36. Boehnke KF, Litinas E, Clauw DJ. Medical cannabis use is associated with decreased opiate medication use in a retrospective cross-sectional survey of patients with chronic pain. J Pain. 2016;17:739-744.

37. Nielsen S, Sabioni $P$, Trigo JM, et al. Opioid-sparing effect of cannabinoids: a systematic review and meta-analysis. Neuropsychopharmacology. 2017;42:1752.

38. Cooper ZD, Bedi G, Ramesh D, et al. Impact of co-administration of oxycodone and smoked cannabis on analgesia and abuse liability. Neuropsychopharmacology. 2018;43:2046-2055.

39. Maguire DR, Yang W, France CP. Interactions between $\mu$-opioid receptor agonists and cannabinoid receptor agonists in rhesus monkeys: antinociception, drug discrimination, and drug self-administration. J Pharmacol Exp Ther. 2013;345:354-362.

40. Hammell DC, Zhang LP, Ma F, et al. Transdermal cannabidiol reduces inflammation and pain-related behaviours in a rat model of arthritis. Eur J Pain. 2016;20:936-948.

41. Harris HM, Sufka KJ, Gul W, et al. Effects of delta-9-tetrahydrocannabinol and cannabidiol on cisplatin-induced neuropathy in mice. Planta Med. 2016;82:1169-1172.

42. Ward SJ, McAllister SD, Kawamura R, et al. Cannabidiol inhibits paclitaxel-induced neuropathic pain through $5-\mathrm{HT}(1 \mathrm{~A})$ receptors without diminishing nervous system function or chemotherapy efficacy. Br J Pharmacol. 2014;171:636-645.

43. Neelakantan $\mathrm{H}$, Tallarida RJ, Reichenbach ZW, et al. Distinct interactions of cannabidiol and morphine in three nociceptive behavioral models in mice. Behav Pharmacol. 2015;26:304-314.

44. Hurd YL, Yoon M, Manini AF, et al. Early phase in the development of cannabidiol as a treatment for addiction: opioid relapse takes initial center stage. Neurotherapeutics. 2015;12:807-815.

45. Laviolette SR. Cannabinoid regulation of opiate motivational processing in the mesolimbic system: the integrative roles of amygdala, prefrontal cortical and ventral hippocampal input pathways. Curr Opin Behav Sci. 2017;13:46-54.

46. Markos JR, Harris HM, Gul W, et al. Effects of cannabidiol on morphine conditioned place preference in mice. Planta Med. 2018;84:221-224.

47. Prud'homme $M$, Cata R, Jutras-Aswad D. Cannabidiol as an intervention for addictive behaviors: a systematic review of the evidence. Subst Abuse Res Treat. 2015;9:33-38.

48. Vigil JM, Stith SS, Adams IM, et al. Associations between medical cannabis and prescription opioid use in chronic pain patients: a preliminary cohort study. PLoS One. 2017;12:e0187795.

49. Bradford AC, Bradford WD. Medical marijuana laws reduce prescription medication use in medicare part D. Health Aff (Millwood). 2016;35: 1230-1236.

50. Livingston MD, Barnett TE, Delcher $C$, et al. Recreational cannabis legalization and opioid-related deaths in Colorado, 2000-2015. Am J Public Health. 2017;107:1827-1829.

51. Campbell G, Hall WD, Peacock A, et al. Effect of cannabis use in people with chronic non-cancer pain prescribed opioids: findings from a 4-year prospective cohort study. Lancet Public Health. 2018;3:e341-e350.

52. Kondrad E, Reid A. Colorado family physicians' attitudes toward medical marijuana. J Am Board Fam Med. 2013;26:52-60.

53. Häuser W, Petzke F, Fitzcharles MA. Efficacy, tolerability and safety of cannabis-based medicines for chronic pain management-an overview of systematic reviews. Eur J Pain. 2018;22:455-470.

54. Bahorik AL, Leibowitz A, Sterling SA, et al. Patterns of marijuana use among psychiatry patients with depression and its impact on recovery. J Affect Disord. 2017;213:168-171.

55. Curran HV, Freeman TP, Mokrysz C, et al. Keep off the grass? Cannabis, cognition and addiction. Nat Rev Neurosci. 2016;17:293-306.

56. Nurmikko TJ, Serpell MG, Hoggart B, et al. Sativex successfully treats neuropathic pain characterised by allodynia: a randomised, double-blind, placebo-controlled clinical trial. Pain. 2007;133:210-220.
57. Ferrè L, Nuara A, Pavan G, et al. Efficacy and safety of nabiximols (Sativex $\left({ }^{\circledR}\right)$ ) on multiple sclerosis spasticity in a real-life Italian monocentric study. Neurol Sci. 2016;37:235-242.

58. Darkovska-Serafimovska M, Serafimovska T, Arsova-Sarafinovska Z, et al. Pharmacotherapeutic considerations for use of cannabinoids to relieve pain in patients with malignant diseases. J Pain Res. 2018;11:837-842.

59. Flachenecker $P$, Henze T, Zettl UK. Nabiximols (THC/CBD oromucosal spray, Sativex ${ }^{\circledR}$ ) in clinical practice-results of a multicenter, non-interventional study (MOVE 2 ) in patients with multiple sclerosis spasticity. Eur Neurol. 2014;71:271-279.

60. Ko GD, Bober SL, Mindra S, et al. Medical cannabis - the Canadian perspective. J Pain Res. 2016;9:735-744.

61. Robson P. Abuse potential and psychoactive effects of $\delta$-9tetrahydrocannabinol and cannabidiol oromucosal spray (Sativex), a new cannabinoid medicine. Expert Opin Drug Saf. 2011;10:675-685.

62. Wade D. Evaluation of the safety and tolerability profile of Sativex ${ }^{\circledR}$ : is it reassuring enough? Expert Rev Neurother. 2012;12(Suppl 4):9-14.

63. Bhattacharyya S, Atakan Z, Martin-Santos R, et al. Neural mechanisms for the cannabinoid modulation of cognition and affect in man: a critical review of neuroimaging studies. Curr Pharm Des. 2012;18:5045-5054.

64. Bidwell LC, Mueller R, YorkWilliams S, et al. A novel observational method for assessing acute responses to cannabis: preliminary validation using legal market strains. Cannabis Cannabinoid Res. 2018;

65. Englund A, Morrison PD, Nottage J, et al. Cannabidiol inhibits THC-elicited paranoid symptoms and hippocampal-dependent memory impairment. J Psychopharmacol. 2013;27:19-27.

66. Hermann D, Schneider M. Potential protective effects of cannabidiol on neuroanatomical alterations in cannabis users and psychosis: a critical review. Curr Pharm Des. 2012;18:4897-4905.

67. Etges T, Karolia K, Grint T, et al. An observational postmarketing safety registry of patients in the UK, Germany, and Switzerland who have been prescribed Sativex ${ }^{\circledR}$ (THC:CBD, nabiximols) oromucosal spray. Ther Clin Risk Manag. 2016;12:1667-1675.

68. Fernández Ó. THC:CBD in daily practice: available data from UK, Germany and Spain. Eur Neurol. 2016;75(Suppl 1):1-3.

69. Karschner EL, Darwin WD, Goodwin RS, et al. Plasma cannabinoid pharmacokinetics following controlled oral $\Delta 9$-tetrahydrocannabinol and oromucosal cannabis extract administration. Clin Chem. 2011;57:66-75.

70. Wang T, Collet J-P, Shapiro S, et al. Adverse effects of medical cannabinoids: a systematic review. Can Med Assoc J. 2008;178:1669-1678.

71. Piper BJ, DeKeuster RM, Beals ML, et al. Substitution of medical cannabis for pharmaceutical agents for pain, anxiety, and sleep. J Psychopharmacol. 2017;31:569-575.

72. Hazekamp A, Ware MA, Muller-Vahl KR, et al. The medicinal use of cannabis and cannabinoids - an international cross-sectional survey on administration forms. J Psychoactive Drugs. 2013;45:199-210.

73. Ware MA, Wang T, Shapiro S, et al. Smoked cannabis for chronic neuropathic pain: a randomized controlled trial. CMAJ. 2010;182:E694-E701.

74. Vandrey R, Raber JC, Raber ME, et al. Cannabinoid dose and label accuracy in edible medical cannabis products. JAMA. 2015;313: 2491-2493.

75. Williams AR, Olfson $\mathrm{M}$, Kim JH, et al. Older, less regulated medical marijuana programs have much greater enrollment rates than newer 'Medicalized' programs. Health Aff (Millwood). 2016;35:480-488.

Cite this article as: Hutchison KE, Hagerty SL, Galinkin J, Bryan AD, Bidwell LC (2019) Cannabinoids, pain, and opioid use reduction: the importance of distilling and disseminating existing data, Cannabis and Cannabinoid Research 4:3, 158-164, DOI: 10.1089/can.2018.0052.

\section{Abbreviations Used}

$\mathrm{CBD}=$ cannabidiol

$\mathrm{RCT}=$ randomized controlled trial

$\mathrm{THC}=\Delta$ 9-tetrahydrocannabinol 